\title{
¿CÓMO FLOTAN LOS CUERPOS QUE FLOTAN? CONCEPCIONES DE LOS ESTUDIANTES
}

\author{
BARRAL, F.M. \\ Departamento de Didáctica das Ciencias Experimentais. Santiago de Compostela.
}

\section{SUMMARY}

A research was carried on pupil's ideas, from Primary School to University, on the flotation of cork, wood and ice on water. Different alternative ideas were found, being the commonest the belief in some bodies floating over the water surface, without any submerged volume. Causes of these alternative conceptions are discussed, and some implications for Science teaching-learning suggested.

«Todo cuerpo sumergido en un líquido experimenta un empuje vertical numéricamente igual al peso del líquido desalojado» (Principio de Arquímedes, 287-212 a.C.).

\section{INTRODUCCIÓN}

Aunque el principio de Arquímedes se estudia tradicionalmente en las clases de Ciencias desde los primeros niveles, suele ser aprendido de forma mecánica o memorística. Es decir, los estudiantes pueden memorizar su estructura gramatical con facilidad, y, por tanto, describirlo verbalmente de forma correcta, mientras que, por el contrario, no es fácil que sea aprendido significativamente. Como muestra la experiencia, y bastantes trabajos de investigación han puesto de manifiesto, las numerosas consecuencias que de este principio se derivan, no son incorporadas a la estructura cognitiva de los estudiantes de forma significativa. Es mucho más fácil que los alumnos se limiten a una mera «asimilacion» de las nuevas ideas científicas -en el sentido que proponen Posner y colaboradores (1982)-que se realice una verdadera "acomodación» de estas ideas, to cual requiere un mayor esfuerzo, al necesitar hacer compati- bles sus creencias con el entendimiento de las nuevas ideas. Creemos que el principio de Arquímedes es un ejemplo paradigmático de este problema:

Prueba a un alumno de $7^{\circ}$ de EGB realizada el 10-3-89:

Pregunta: ¿Por qué crees que se infla un balón al introducir aire en su interior?

Contestación: Porque todo cuerpo sumergido en un fluido experimenta un empuje vertical y hacia arriba igual al peso del fluido que desaloja.

En el campo de la estática de fluidos y más concretamente sobre el problema de la flotación han aparecido en la bibliografía algunos estudios. Bien dentro de la línea evolutiva (Inhelder y Piaget 1985), trabajos que trataban el problema de la flotación pero que eran realizados con 
el objetivo de estudiar los estadios de desarrollo evolutivo, o bien trabajos dentro de la línea constructivista del aprendizaje, como el estudio sistemático llevado a cabo dentro del proyecto LISP(P) de la Universidad de Waikato (Osborne 1983, Biddulph 1983, Biddulph y Osborne 1984, Symington 1983, Appieton y col. 1984, Biddulph y col. 1984) y trabajos más puntuales como los de Carretero (1979), Guyon y Misery (1980), Dentici y col. (1984), Fernández Fernández $(1985,1987)$, Mullet $\mathrm{y}$ Montcouquiol (1988)...

El concepto clave para entender la flotación es sin duda el empuje, y la mayoría de los trabajos que se han realizado hasta el momento sobre las concepciones de los alumnos se centran en estudiar de qué variables creen los alumnos que depende el empuje (masa o peso del cuerpo, volumen, forma, densidad del fluido...). Pero previamente a dicho problema está el de la misma existencia del empuje. Al ser el empuje una fuerza ino tendrán los alumnos dificultades asociadas con las que se derivan de la misma naturaleza de este concepto? Como demuestran los estudios llevados a cabo en el campo de la mecánica acerca del concepto de fuerza, las concepciones intuitivas que poseen los alumnos son variadas y muy difíciles de cambiar. En relación a este aspecto, el trabajo de Guyon y Misery (1980) ya aporta una idea interesante: algunos alumnos creen que «si los objetos se hunden es porque no existe el empujem. Queda por investigar lo que creen los alumnos acerca de los objetos que flotan. $Y$ es en este aspecto, en el que se centra el estudio que se describe en este trabajo.

\section{DESCRIPCIÓN DEL ESTUDIO REALIZADO}

La prueba elegida para conocer las concepciones de los alumnos sobre cómo flotan en el agua los cuerpos que flotan, consistió en pedirles que dibujasen trozos de corcho, madera y hielo flotando en agua. Nos interesaba que el esquema reflejara tanto la parte sumergida del objeto como la parte que quedaba por encima del agua, por ello se les indicaba que dibujasen un recipiente de agua como el representado en la figura 1 y los objetos, tanto el corcho, la madera como el hielo de forma rectangular para facilitar la comparación.

La realización de dibujos como método de recogida de datos es, como indican De Vecchi y Giordan (1988), una de las vías importantes para poder establecer las ideas de los alumnos, ya que se corresponde con un modo de expresión muy adaptado a nuestra época y en ocasiones más accesible que el verbal, pues muchos alumnos poseen desde muy jóvenes la capacidad para realizar esquemas. AI mismo tiempo, y para saber si los esquemas representaban exactamente lo que pensaban los alumnos y no que fueran «una forma de đibujar», en casos donde los dibujos representaban concepciones alternativas, se realizaron entrevistas pidiéndoles explícitamente que nos explicaran por qué habían hecho los dibujos de los objetos flotando de esa forma. figura 1

Recipiente de agua y objeto rectangular propuestos para que los afumnos realizaran los dibujos.

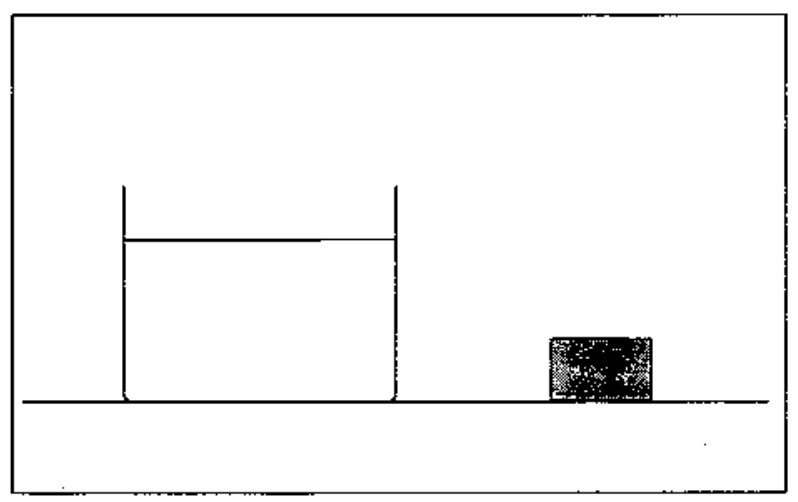

\section{Muestra de alumnos}

Para disponer de datos correspondientes a un amplio abanico de niveles de enseñanza, de Primaria hasta la Universidad, se realizó la recogida de datos con tres grupos ( $N=108$ ) de colegios distintos de 8 curso de EGB (13-14 años); un grupo $(\mathrm{N}=26)$ de 20 curso de BUP (1516 años); un grupo de COU $(\mathrm{N}=19)$ y dos grupos de alumnos de Escuela Universitaria del Profesorado de EGB de Santiago, uno $(\mathrm{N}=36)$ de segundo curso de la especialidad de Ciencias (todos estos alumnos habían cursado la asignatura de Física en COU) y el otro grupo $(\mathrm{N}=39)$ de tercer curso de la especialidad de preescolar (en el que la mayoría no habían cursado la Física de COU ni la Física y Química de $3^{\circ}$ de BUP). Todos los centros pertenecen a la provincia de La Coruña.

\section{Método de análisis}

Las densidades de las sustancias empleadas en la prueba se recogen en la Tabla I.

Tabla I

Densidades de los sólidos utilizados para la prueba (tornadas del Handbook of Chemistry and Physics, 61 Ed., I980-81, CRC Press, Florida) 
Los alumnos, al no disponer de los datos de densidad de las sustancias, debían empiear sus propios datos. Esperábamos, que en líneas generales, pensaran que el corcho era el más ligero de los cuerpos, seguido de la madera (como se aprecia en los datos de la Tabla I las maderas más conocidas tienen una densidad intermedia entre el corcho y el hielo, no creemos que ningún alumno piense en tipos de madera, como el ébano, más densas que el agua), y del hielo.

No nos interesaba en esta investigación, la fracción đe cuerpo que dejaban fuera del agua frente a la que dibujaban dentro, sino que clasificamos en la misma categoría todos los esquemas que representaban los cuerpos con una parte fuera del agua y otra dentro, cualquiera que fuera la proporción entre ellas.

El análisis de los esquemas realizados por los alumnos de Ios diferentes grupos dio como resultado una gran variedad de categorías que se recogen en la figura 2 .

\section{figura 2}

Esquemas que representan las concepciones de los alumnos acerca de la flotación del corcho, madera y hielo en agua.

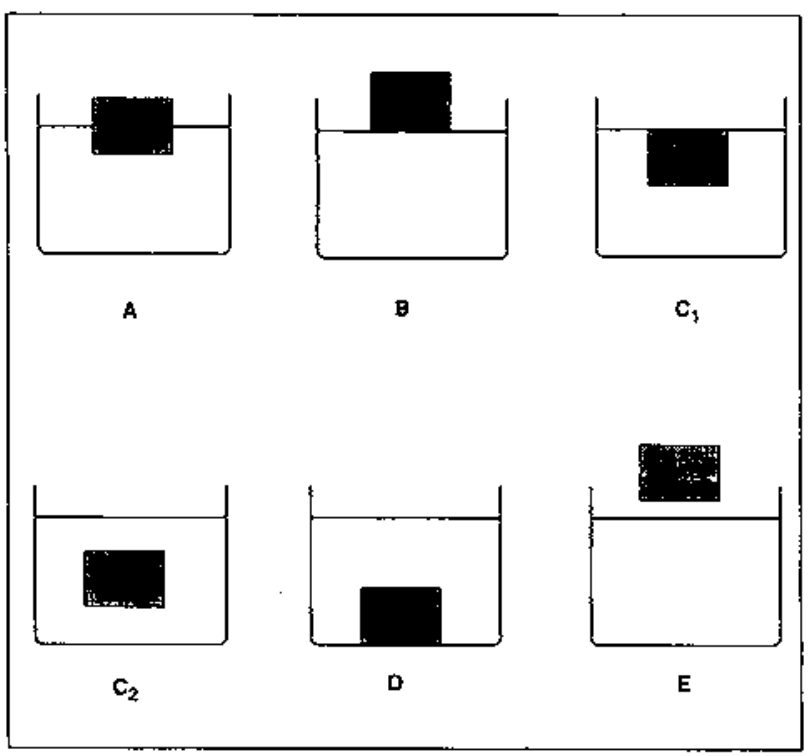

El esquema A, considerado como correcto, simboliza la categoría de concepciones de alumnos que representan los cuerpos flotando en el agua con parte sumergida y parte fuera, no importando, como se dijo en el apartado anterior, la proporción entre las dos partes.

El esquema $B$, representa la categoría de alumnos que dibujan el objeto totalmente fuera del agua aunque en contacto con ella.

Los esquemas $\mathrm{C}_{4}, \mathrm{C}_{2}$ y D, representan los dibujos de los alumnos que colocan los objetos totalmente introducidos en el agua; el $\mathrm{C}^{1}$ con el objeto en contacto con la superficie, el $\mathrm{C}_{2}$ con el objeto «entre dos aguas» y el D en el fondo.
Por último el esquema E corresponde a una concepción muy minoritaria, de aquellos alumnos que dibujan algunos de los objetos totalmente fuera del agua sin tocarla siquiera.

Por otro lado, clasificamos como incodificables (I) los esquemas de aquellos alumnos que realizaban dibujos que no permitían establecer claramente la categoría a la que correspondían. En general estos dibujos corresponden a vistas desde arriba o con la superficie del agua en forma de ondas, de forma que no se puede establecer hasta qué punto está sumergido el objeto.

\section{RESULTADOS Y ANÁLISIS DE LAS CON- CEPCIONES DE LOS ESTUDIANTES}

En la Tabla II, se recogen los resultados por número de alumnos.

Tabla II

Concepciones de los alumnos sobre la flotación del corcho (C), madera (M) y hielo (H) en agua.

\begin{tabular}{|c|c|c|c|c|c|c|c|c|}
\hline \multicolumn{2}{|c|}{ CATEGORIAS } & A & B & $c_{1}$ & $\mathrm{c}_{2}$ & D & E & 1 \\
\hline \multicolumn{9}{|c|}{ N' ALUMNOS } \\
\hline & $\mathrm{C}$ & 44 & 59 & $\cdot$ & 1 & $\cdot$ & - & 4 \\
\hline \multirow[t]{3}{*}{$8^{\circ} E G B$} & $M$ & 72 & 21 & 6 & 2 & 1 & $\cdot$ & 6 \\
\hline & $\mathrm{H}$ & 46 & 8 & 25 & 10 & 15 & $\cdot$ & 4 \\
\hline & $c$ & 10 & 16 & . & - & - & - & . \\
\hline \multirow[t]{3}{*}{$2^{\circ}$ BUP } & $M$ & 24 & 1 & $\mathfrak{\imath}$ & - & - & - & - \\
\hline & $H$ & 14 & 1 & 3 & 4 & 4 & - & - \\
\hline & $\mathrm{c}$ & 4 & 13 & 1 & $\cdot$ & $\cdot$ & 1 & - \\
\hline \multirow[t]{3}{*}{$\mathrm{cou}$} & $M$ & 15 & 1 & 2 & $\cdot$ & . & 1 & - \\
\hline & $\mathrm{H}$ & 8 & 1 & 4 & $\cdot$ & 5 & 1 & . \\
\hline & $c$ & 14 & 24 & - & - & $\cdot$ & 1 & . \\
\hline \multirow[t]{3}{*}{ अ E.U. } & $M$ & 27 & 8 & 1 & 1 & - & 1 & 1 \\
\hline & H & 24 & $\mathrm{~s}$ & 3 & 3 & 3 & - & i \\
\hline & c & 21 & in & . & 1 & 1 & $\cdot$ & 2 \\
\hline \multirow[t]{2}{*}{$2^{0} \varepsilon . u}$. & $M$ & 28 & 7 & - & . & - & . & 1 \\
\hline & $\mathrm{H}$ & 34 & $\cdot$ & $\cdot$ & 2 & $\cdot$ & $\cdot$ & - \\
\hline
\end{tabular}

\section{La concepción: «flota totalmente»}

De los resultados anteriores, destaca el elevado número de alumnos que presentan en sus dibujos la concepción representada por el esquema B (fig. 2), concepción que es mayoritaria para el caso del corcho y no despreciable para la madera o el hielo.

El que esta interpretación no coincida con la científica así como que se mantenga después de varios períodos de 
instrucción (fig. 3), nos hace pensar que se trata de una verdadera concepción alternativa.

\section{figura 3}

Concepciones de los alumnos sobre la flotación del corcho.

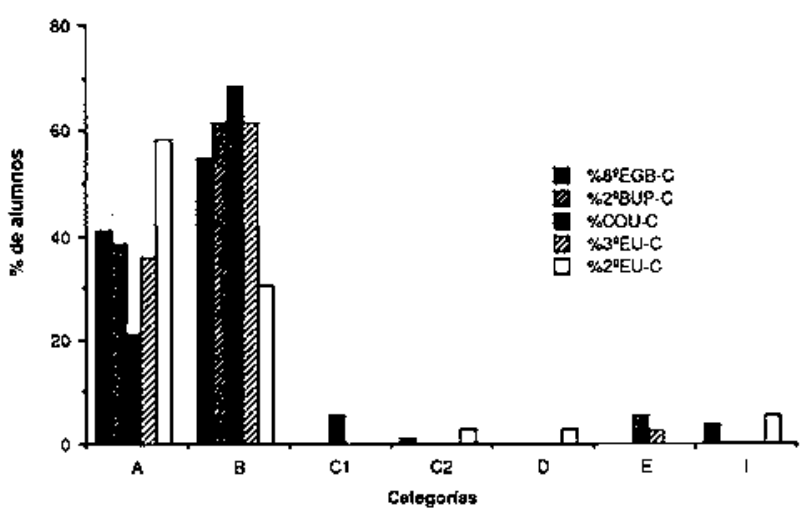

De las entrevistas realizadas con alumnos que presentan esta concepción, se desprende que sus dibujos están de acuerdo con sus ideas. Como ejemplo podemos transcribir algunas de las explicaciones:

«La madera flota por encima del agua» (alumno de EGB que dibujó la madera según el esquema $B$ ).

«El taco de corcho flota en la superficie del agua» $\left(2^{\circ}\right.$ de BUP).

«El hielo está parcialmente sumergido, la madera también pero el corcho flota totalmente» (COU).

«El corcho flota en la superfície sin hundirse en el agua» (COU).

«El corcho flota el $100 \%$ y la madera el $50 \%$ ( $3^{2} \mathrm{de}$ E.U.).

«El taco de corcho va a quedar en la superficie del agua, es decir, quedará rozando justo con la superficie del agua, ya que su peso es menor que el empuje que va a ejercer el agua hacia arriba» ( $2^{\circ}$ de E.U.).

«La densidad de la madera es mayor que la densidad del corcho y por tanto éste quedará completamente encima de la superfície del agua, mientras que la madera quedará un poco sumergida» ( $2^{\circ}$ de E.U.).

Está claro que el esquema B representa una concepción de la flotación muy extendida entre los alumnos; existen cuerpos - como el corcho- que flotan totalmente.

Ningún alumno hizo mención a la tensión superficial que podría justificar en algún caso la creencia en esta concepción.
Esta concepción alternativa se refleja también en los trabajos de Inhelder y Piaget (1985) a los que nos referíamos antes. La transcripción de alguna de las frases que los niños emplean en sus entrevistas así to puede sugerir (los subrayados son nuestros):

Iea (4 años), dice acerca de un pedazo de madera: «Permanece encima, el otro día tiré un pedazo al agua y se quedó encima».

Duf $(7 ; 6):$ «Esta bola? Permanece sobre el agua; es madera, es liviana»

Ahora bien, ¿cuál puede ser el origen u orígenes de esta concepción?

Como en la mayoría de las concepciones alternativas, las experiencias sensoriales juegan un papel muy importante (Driver y Erickson 1983). La flotación de los cuerpos es un fenómeno con el que estamos muy familiarizados desde pequeños, del que tenemos numerosos registros sensoriales sin haber reflexionado sobre el conjunto del problema. Como la mayor parte de los objetos que vemos flotando los vemos desde arriba, en una perspectiva que no permite apreciar fácilmente la parte sumergida del objeto, puede resultar plausible tener la impresión -sobre todo en el caso del corcho-de que «flota totalmente».

Por otro lado el uso de la preposición «sobre» en el lenguaje ordinario cuando se trata con problemas de flotación es muy frecuente: «el hielo flota sobre el agua», lo que puede reforzar la concepción que venimos estudiando. Para algunos alumnos no está muy claro lo que significa la palabra «flotando». En el trabajo de Biddulph (1983) en el que se investiga con un dibujo de un iceberg si creen que flota o no, alguna respuesta es significativa en este sentido. Hay niños que creen que el icerberg flota y no flota al mismo tiempo por tener parte sumergida y parte no sumergida:

«It's floating and not floating. The top is floating and the bottom's not»

La persistencia de esta concepción después de la instrucción en estática de fluidos, y en concreto del principio de Arquímedes, en varios niveles de la enseñanza, es un hecho que se puede analizar con mayor profundidad. La primera conclusión a este respecto, es la de que no existe un aprendizaje significativo de dicho principio. No se aprecia la necesidad de la existencia del empuje para que los objetos floten ni por tanto la de que exista un volumen sumergido de cualquier cuerpo que flote en el agua. Existe una concepción alternativa bien conocida en mecánica que ha sido investigada por numerosos autores (Clement 1982, McDermott 1984) y que se puede resumir en que los alumnos creen que «tiene que haber fuerzas actuando sobre los cuerpos en movimiento, en la dirección de dicho movimiento, y no existen fuerzas actuando sobre los cuerpos en equilibriom. Como cuando los cuerpos están flotando existe equilibrio, se puede creer que no existen fuerzas actuando sobre ellos y por tanto al no haber empuje, no tiene por qué haber volumen sumergido. 
En el caso del corcho y el agua y, dentro del conflicto que juegan sólido y líquido en los problemas de flotación (Mullet y Montcouquiol 1988), es posible que los alum. nos den un papel demasiado pasivo tanto a uno como al otro, o bien solamente papel activo al agua que «sostiene el corcho en la superficie».

Parece claro que cuando un cuerpo que flota está total. mente sumergido y en movimiento hacia la superficie (el corcho por ejemplo) los alumnos ya instruidos piensan en la existencia del empuje, fuerza que deja de actuar cuando el cuerpo llega a la superficie en donde no se piensa que sea necesario seguir contrarrestando el peso del objeto ( $i$ el corcho pesa tan poco!). Piaget e Inbelder (1982) encuentran que los niños explican el aumento del nivel del agua por immersión de un sólido en ella, más como debido al peso del objeto que como el espacio ocupado por el sólido. Este problema está asociado al de que «aparentemente» un cuerpo que flota no pesa nada (el đinamómetro marcará cero).

De todas formas creemos que la persistencia de esta concepción es debida no tanto, a una «resistencia al cambio", como a una ausencia de estrategias adecuadas en las pautas de instrucción para que este cambio se produzca.

\section{Las concepciones: «totalmente sumergido» y «se va al fondo»}

Aunque menos extendida que la concepción que estudia. mos en el apartado anterior, existe un buen número de alumnos que (sobre todo para el caso del hielo y en menor medida para la madera) presentan otras concep. ciones alternativas representadas por las categorías $C_{1}, C_{2}$, y D. Dibujan los trozos de hielo o madera totalmente sumergidos en el agua, bien en contacto con la superficie $\left(C_{1}\right)$ o bien entre dos aguas $\left(C_{2}\right)$ o incluso piensan que se van al fondo (D) (figura 4).

\section{figura 4}

Concepciones de los alumnos sobre la flotación del hielo.

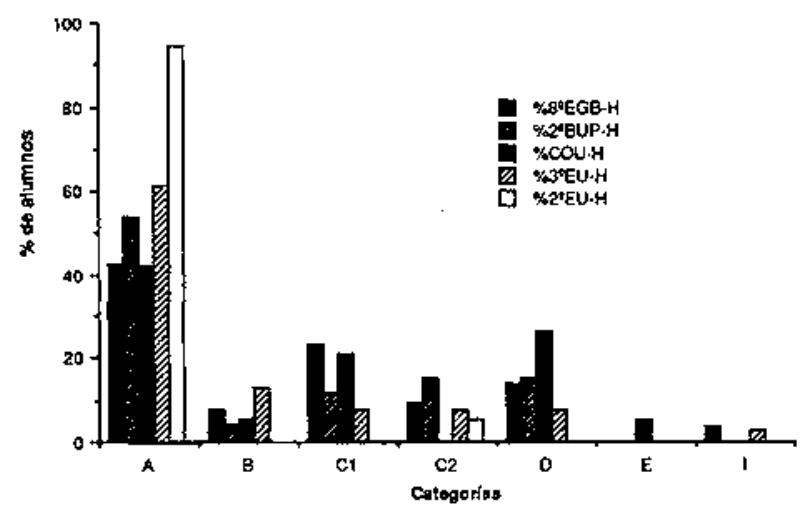

De nuevo, las experiencias sensoriales parecen jugar un papel importante en la existencia de estas concepciones alternativas. Algunos alumnos dibujan varios cubos de hielo totalmente sumergidos, unos encima de otros. La tendencia a pensar en los vasos de refresco con cubitos de hielo es muy fuerte: algunos alumnos convierten el esquema que se les pedía en un vaso de refresco con «pajita» y todo.

Otros alumnos dicen que el hielo «se va al fondo" porque se derrite:

«El hielo se hunde, poco a poco se va derritiendo» $\left(8^{\circ} \mathrm{de}\right.$ EGB).

Algunos dibujan un trozo de hielo con flechas hacia abajo o con líneas hasta el fondo del recipiente para indicar cómo el hielo no va a permanecer cerca de la superficie durante mucho tiempo: piensan en la fusión del hielo en agua que se va a terminar confundiendo con ella.

Quizá a diferencia del esquema «flota totalmente», en este caso - hielo flotando en agua- los alumnos dan un papel demasiado activo al sólido y demasiado pasivo al líquido (Mullet y Montcouquiol 1988).

\section{IMPLICACIONES PARA LA ENSEÑANZA}

Uno de los principales objetivos de la enseñanza de las ciencias, en cualquier nivel, es el ayudar a los alumnos a tener una visión que dé un mayor sentido al mundo en el que viven, proporcionarles nuevas perspectivas que sean más fructíferas que sus ideas anteriores. $\mathrm{Al}$ mismo tiempo ayudaremos al alumno a reconocer que muchas ideas científicas son de una utilidad inestimable para la sociedad. Más que en la dirección de hacer futuros científicos o técnicos, sería para que las personas encuentren individualmente que las ideas a las que los acercamos en las clases de ciencias, son ideas inteligibles, plausibles y útiles (Osborne 1985). Entre otros hechos de la vida cotidiana que pueden ser ejemplo de esto, se encuentra el problema de la flotación de los cuerpos --por ejemplo el nivel de flotación del cubo de hielo en nuestro refrescom resuelto historicamente por Arquímedes.

Parece claro también que en numerosas ocasiones, nuestras experiencias de la vida cotidiana no nos proporcionan la información suficiente para que cada uno de nosotros comprenda nuestro entorno inmediato. $Y$ que incluso después de recibir más información en las clases de ciencias, seguimos sin comprenderlo de forma adecuada. El aprendizaje memorístico y a veces de forma muy superficial (Carrascosa y Gil 1985) de las ideas científicas que se enseñan en la escuela llevan a situaciones como las encontradas en esta investigación.

No nos debemos engañar. Tođavía existen muchos profesores y diseñadores de currículos que no aprecian la 
importancia de la existencia de las ideas de los alumnos y su resistencia al cambio. Por ello, no es sorprendente que las concepciones de los alumnos, frecuentemente permanezcan inalteradas o que se modifican en aspectos no esperados.

Si queremos que esta situación cambíe, debemos adoptar en nuestras clases de ciencias estrategias que tengan en cuenta las ideas de los alumnos (Giordan 1989). Los profesores de ciencias, tendrán que ser conscientes de las dificultades que van a tener sus alumnos en el aprendizaje de determinados conceptos, principios o modelos para poder plantear métodos de enseñanza basados en esta comprension. Y son las investigaciones sobre las ideas alternativas de los alumnos las que proporcionan dicha información al profesor, que envuelto en el complejo mundo de una clase de ciencias con todas sus situaciones particulares, posiblemente necesite ayuda en este sentido. Por ello los materiales para los profesores deberían proporcionar información sobre las ideas de los alumnos -lo que raramente hacen- ya que éstas influirán en el aprendizaje de una situación o tema particular.

El profesor se encontrará en mejor posición para tomar decisiones pedagógicas de acuerdo con estos datos y realizar aproximaciones más fructíferas al proceso de enseñanza-aprendizaje en temas concretos.

La utilización de estrategias basadas en perspectivas de tipo constructivista parece la solución más adecuada si queremos que la enseñanza de las ciencias mejore. No

\section{REFERENCIAS BIBLIOGRÁFICAS}

APPLETON, K., HAWE, E., BIDDULPH, F., HAMILL, E. y GREGAN, P, 1984. Floating and Sinking: First teacher trials, Working Paper $N^{2} 120$, Learning in Science Project (Primary), Science Education Research Unit, University of Waikato, N.Z.

BIDDULPH, F., 1983. Students' Views of Floating and Sinking, Working Paper $\mathrm{N}^{*} 116$, Learning in Science Project (Primary), Science Education Research Unit, University of Waikato, N.Z.

BIDDULPY, F. y OSBORNE, R, 1984. Floating and Sinking: Trial Units, Working Paper $N^{2} 117$, Learning in Science Project (Primary), Science Education Research Unit, University of Waikato, N.Z.

BIDDULPH, F., APPLETON, K., FAIRE, J., DUNCAN, J. y ROGER, J., 1984. Floating and Sinking: Second teacher trials, Working Paper $N^{2} 121$, Learning in Science Project (Primary), Science Education Research Unit, University of Waikato, N.Z. cabe duda de que tanto por parte del alumno (mucho mayor esfuerzo de reflexión, comunicación de sus ideas...) como por parte del profesor (¿cuántas veces en las clases estamos realmente interesados en esas ideas «no aceptables» que mantienen los alumnos?, ¿tenemos una tendencia natural a sintonizar «selectivamente» solamente con aquellas respuestas de alumnos que estamos buscando?) este tipo de estrategias requerirán mayor compromiso (tiempo dedicado a un determinado tema, discusiones en pequeño y gran grupo...) y trabajo más intenso (currículo en continua revisión de acuerdo con los resultados obtenidos, cambio en la evaluación...) pero sin duda también se verán gratificados por mejores resuitados.

\section{AGRADECIMIENTOS}

El autor quiere agradecer a la Comisión Interdepartamental de Ciencia e Tecnoloxía de Galicia la concesión de la «Axuda para o fomento da investigación científica e técnica»: XUGA71804688, para la realizacion del Proyecto AcAb de investigación educativa, dentro del cual se enmarca la investigación que se recoge en el presente artículo.

A los profesores J. Ramón Gallástegui Otero, Mâ Dolores Seijo Pena, Mercedes López García y M ${ }^{3}$ Carmen Suárez Calvo, la colaboración prestada, ya que sin su ayuda este trabajo no habría sido posible.
CARRASCOSA, J. y GIL, D., 1985. La «metodología de la superficialidad" y el aprendizaje de las ciencias, Enseñanza de las Ciencias, Vol. 3, pp. 113-120.

CARRETERO, M., 1979. ¿Por qué flotan las cosas? El desamolto del pensamiento hipotético-deductivo y la ensenanza de la ciencia, Infancia y Aprendizaje, 8, pp.7-22.

CLEMENT, J., 1982. Students' preconceptions in introductory mechanics, American Journal of Physics, Vol. 50, pp. 6671 .

DE VECCHI, G. y GIORDAN, A., 1988. L'Enseignement Scientifique: Comment faire pour que ça "marche"?, Guides practiques ( Z'editions: Niza).

DENTICI, O.A., GROSSI, M.G., BORGHI, L., DE AMBROSIS, A. y MASSARA, C.I, 1984. Understanding floating: A study of children aged between six and eight years, European Journal of Science Education, Vol. 6, pp. 235-243. 
DRIVER, R. y ERICKSON, G., 1983. Theories-in-Action: Some Theoretical and Empirical Issues in the Study of Students' Conceptual Frameworks in Science, Studies in Science Education, Vol. 10, pp. 37-60.

FERNÁNDEZ FERNÁNDEZ, J.M., 1985. Causas de las difí cultades de aplicación del Teorema de Arquímedes por los alumnos de enseñanza media, Enseñanza de las Ciencias, Vol. 3. pp. 185-187.

FERNÁNDEZ FERNÁNDEZ, J.M., 1987. Estudio del grado de persistencia de ciertos preconceptos sobre la Estática de Fluidos en alumnos del $2^{2}$ curso del BUP, Enseñanza de las Ciencias, Vol. 5, pp. 27-32.

GIORDAN, A., 1989. Representaciones sobre la utilización didáctica de las representaciones, Enseñanza de las Ciencias, Vol. 7, pp. 53+62.

GUYON, J. y MISERY, M., 1980. Travaux relatifs a une recherche concernant le volume, la masse volumique et la poussée D'Archimède en classe de cinquième, ASTER: Recherches Pedagogiques, 18, pp. 117-122.

MULLET, E. y MONTCOUQUIOL, A., 1988. Archimedes'Effect, information integration and individual differences, Inter- national Journal of Science Education, Vol. 10, pp. 285301.

MCDERMOTT, L.C., 1984. Research on conceptual understanding in mechanics, Physics Today, Vol. 37, pp. 24-32.

OSBORNE, R., 1983. Video: floating and sinking, Working Paper N ${ }^{8} 115$, Learning in Science Project (Primary), Science Education Research Unit, University of Waikato, N.Z.

OSBORNE, R., 1985. Children's own concepts, en Wynne Harlen (ed.), Primary Science... Taking the Plunge (Heinemann: Oxford), pp. 75-91.

PIAGET, J. E INHELDER, B., 1982. El desarrollo de las cantidades en el niño. (Hogar del Libro: Barcelona).

POSNER, G.J., STRIKE, K.A., HEWSON, P.W. y GERTZOG, W.A., 1982. Accommodation of a Scientific Conception: Toward a Theory of Conceptual Change, Science Education, Vol. 62, pp. 21 I-227.

SYMINGTON, D, 1983. An Analysis of the LISP(P) Unit Floating and Sinking, Working Paper $N^{2} 118$, Learning in Science Project (Primary), Science Education Research Unit, University of Waikato, N.Z. 